\title{
Endeudamiento externo y crecimiento economico: un análisis de la evidencia empírica
}

\author{
Luis René Cáceres*
}

\section{Introducción}

Los graves problemas de endeudamiento externo enfrentados por los paises latinoamericanos en la década recien pasada obligan a examinar el papel que los recursos exlernos desempenan en el desarrollo económico. Lo anterior cobra urgencia al tomar en cuenta el cúmulo de Irabajos teóricos y empíricos que fueron preparados en las décadas pasadas sobre el papel propulsor que el ahorro externo tendría en la inversión y crecimiento económico. Sin embargo, la realidad de los últimos años muestra la coincidencia de un acelerado endeudamiento con estancamiento económico, a la vez que han surgido los problemas de servicio de la deuda que no hablan sido lomados en cuenta en los modelos tradicionales. Asl, surge la necesidad de precisar la relación entre endeudamiento y crecimiento y. de identificar los condicionantes que determinan el papel beneficioso o negativo de los tondos externos. En este trabajo se analiza el papel del endeudamiento externo en el crecimienlo económico, presentando una reseńa de algunos modelos propuestos sobre recursos externos y crecimiento.

- El aulor es funcionario del Banco Interamericano de Desarrollo.

Los puntos de vista expuesios en este documento son personales. 


\section{Recursos externos, ahorro doméstlco y crecimiento económico}

El modelo tradicional sobre el impacto de los recursos externos en el crecimiento es el modelo de las dos brechas, el cual, en terminos generales, se lormula en base a la identidad $Y=C+I+E-M$, en donde $Y=$ producto nacional bruto; $C=$ consumo público y privado; $I=$ inversión; $E$ = exportaciones: $M$ = importaciones'. A partir de la idenlidad anterior se puede resolver por la inversión, asi:

$$
\mathrm{I}=\mathrm{S}+\mathrm{F}
$$

en donde $F=M-E$ es el monto de recursos externos requeridos para permilir incremenlar los recursos nacionales $Y-C(S)$, hasta alcanzar la cuantia de inversión deseada. En esle caso los recursos exlernos se conceplualizan como un medio para cerrar la brecha de ahorro que ocurre al ser $\mathrm{S}$ inferior a $\mathrm{I}$.

La identidad $F=M-E$ se puede expresar también de la forma:

$$
M=E+F
$$

lo que indica que los recursos externos desempenan la función de cerrar la brecha externa que ocurre cuando las exportaciones no cubren las imporlaciones requeridas para lograr un determinado programa de inversión.

Las identidades anteriores se pueden poner en una forma operativa tomando como base el conocido modelo de Harrod: $\mathbf{g}=\mathbf{s k}$, en donde $\mathrm{g}$ es la lasa de crecimiento del ingreso nacional, $s$ es la propensión marginal de ahorrar y $\mathrm{k}$ es la lasa incremental producto-capilal. Si el pais en cuestión recibe una inyección de recursos externos por $\mathrm{I}$, expresada en términos del ingreso nacional, la lasa de crecimiento es dada ahora por $g=k(s+f)$. De esta manera, si la lasa deseada de crecimiento luese $g^{*}$, el ahorro requerido para lograr esla tasa seria $g^{*} / k=s^{*}$, y la brecha de ahorro seria igual a $s^{*}-s=1$. De la misma manera, se puede representar la brecha externa como $f=m-E N$, en donde $m$ es la propensión marginal a importar.

El modelo anterior tiene el atraclivo de ser sumanente eficaz para fines de cuantificar los recursos exlernos requeridos para sustentar un programa de inversión o para alcanzar una tasa de crecimiento deseada; es útil, además, para ofrecer una expresión lormal a la problemática de los desequilibrios interno y exlemo que con mucha insistencia ha sido presentada como una condición subyacente al subdesarrollo ${ }^{2}$. Sin embargo, el modelo ha sido blanco de muchas objeciones. Una de sus 
principales limilaciones, según sus críticos, es que no permite ninguna flexibilidad al interior de la economía para la sustitución de imporlaciones por producción interna; asimismo, no toma en cuenta que el consumo aclual puede ser disminuido para incrementar el ahorro corriente y asi reducir la brecha de ahorro.

Es con relación al ahorro doméstico que el modelo ha sido más cues. tionado, particularmente por el supuesto de que los recursos externos en su totalidad son destinados a la inversión, sin que ocurra ningún efecto sobre el consumo. La exislencia de un efecto positivo de los recursos externos sobre el consumo ha sido postulado por varios críticos y se han sefialado distintos canales por medio de los cuales se materializa este efecto. Por una parte, el ahorro público puede disminuir como resultado de una merma en la recaudación de impuestos debido a que, ante el ingreso de ayuda externa, el gobierno puede realizar un menor esluerzo para caplar ingresos fiscales. Por su parte, el ahorro del sector privado puede mermar ante la oferta de recursos externos relativamente baralos que reducen el incentivo a ahorrar de parte de los inversionistas; lambién se ha sef̂alado que la tácil disponibilidad de ayuda externa puede conducir al gobierno a reducir o posponer esiuerzos para ampliar la base exportadora y a mantener políticas que lacilitan las importaciones.

La existencia de un efecto negalivo de los recursos externos sobre el ahorro doméstico ha sido delectado en varios estudios. Por ejemplo, Griftin usando una muestra transversal de 36 paises en vias de desarrollo y con datos para el período 1962-1964 encontró los siguientes resultados: ${ }^{3}$

$$
\frac{S}{Y}=11.2-\underset{(0.11)}{0.73} \frac{A}{Y} \quad R^{2}=0.54
$$

en donde $\frac{S}{Y}=$ tasa de ahorro bruto doméstico y $-\frac{A}{Y}=$ ahorro externo como porcentaje del producto interno bruto, y la desviación estándar del coeficiente de $\frac{A}{Y}$ se muestra debajo del mismo. Esle resultado indica que cerca de tres cuartas partes del ahorro externo se destinarian al consumo.

En un trabajo subsecuente Griffin y Enos examinan la relación inversa entre crecimiento económico y ahorro externo en 12 paises latinoamericanos para el período 1857-64 y concluyen que: "... la ayuda exter- 
na no ha acelerado el crecimiento ni ha promovido regimenes pollticos democráticos. La ayuda puede haber retardado el desarrollo al conducir a tasas de ahorro domésticas más bajas, al distorsionar la composición de la inversión, aumentando asi la razón capital-producto, al frustrar la emergencia de una clase empresarial propia $y$ al inhibir retormas instilucionales"3.

Por otra parte, Rahman, usando dalos de 31 palses en vias de desarrollo para el año de 1962, estimó la ecuación siguiente:

$$
S+0.14-\underset{(2.5)}{-0.25} \frac{A}{Y}
$$

La ecuación anterior muestra que una cuarta parte del ahorro externo seria destinada al consumo.

En un trabajo que utilizó una muestra transversal de 17 paises y usando datos del periodo 1953-1965, Weisskopf estimó la siguente ecuación.

$$
S-a+0.183 Y-0.227 F+0.176 E
$$

en donde a representa la ordenada en el origen que varía de pais a pais según el valor de la variable cualitativa estimada, $Y$ es el producto interno brulo, $F$ es el ingreso del capital extranjero y $E$ denota las exportaciones. Se puede notar que aproximadamenle una cuarta parte del ahorro externo se destina al consumo ${ }^{6}$.

Asimismo, se trae a cuenla el estudio de corté transversal de Landau para 18 paises latinoamericanos, usando datos anuales para el periodo 1950-1966, en el que obtuvo la siguiente ecuación:

$$
\frac{S}{Y}=a+\underset{(3.5)}{0.4761} \log \left(\frac{Y}{P}\right)-\underset{(11.8)}{0.5345}\left(\frac{F}{Y}\right) R^{2}=0.80
$$

en donde a es la ordenada en el origen que corresponde a cada pais, $\mathrm{P}$ es la población y $\underset{-\mathrm{Y}}{\mathrm{Y}}$ es el ingreso per cápita.

De acuerdo a esle resultado, la mitad del ahorro externo per cápila se deslinaria al consumo en los paises latinoamericanos ${ }^{7}$. Landau lambién estimó ecuaciones de la forma $S=a+b Y+C F$ para cada 
uno de los 18 pises y en 15 de ellos encontro que los recursos extemos ejercian una ińluencia negativa sobre el ahorro. En los paises en los cuales el coeficiente de $F$ fue posilivo, éste resultó no ser estadisticamente signiticalivo"

En un trabajo sobre los requerimientos de capilal extranjero de los paises latinoamericanos para el periodo 1970-76, Chenery y Eckslein eslimaron funciones de ahorro para 16 paises y encontraron que en 12 de ellos el impacto del capital extranjero era negalivo".

Asimismo, en un estudio para los países centroamericanos, usando dalos del período 1960-1980. Cáceres enconlró que el ahorro externo ejerce un efecto negativo sobre el ahorro doméstico de lodos los paises, excepto Honduras ${ }^{10}$.

En un estudio sobre el papel desempenado por el ahorro externo en el crecimiento económico de Corea, Voivodas estimó una ecuación para el periodo 1962-1968, expresando la lasa de ahorro doméstico, $s$, en función del ahorro exlerno como fracción del produclo inlerno bruto, $\mathbf{f}$, encontrando los resullados siguientes:

$$
\mathrm{S}=1.02+1.321 \quad \mathrm{R}^{2}=0.69 \quad \text { D.W. }=2.15
$$

Sin embargo, para el periodo 1954-1962 la ecuación estimada lue la siguiente:

$$
s=4.34+0.07 f
$$$$
\mathrm{R}^{2}=0.00 \quad \text { D.W. }=1.94
$$

El autor explicó la diferencia en el significado estadistico de la variable $f$ por el hecho de que en la década de los cincuerila la ayuda oficial predominó en los ingresos de capilal, mientras que en la década subsiguiente el ingreso de capital privacto representó el principal componente de dicha variable". De esa manera, sería el flujo de capilal privado extranjero lo que aumentaria la tasa de ahorro.

Se trae a cuenta, además, el trabajo de Gupla, quien estimó, para una muestra de 40 países en vias de desarrollo y usando datos de los anos setenta, una ecuación expresado la tasa de ahorro $\left(\frac{S}{Y}\right)$ en función del ingreso real per cápita $(Y)$, la tasa de crecimiento económico (G), el ingreso del capilal extranjero (F) y la lasa de dependientes (DR), delinida ésta como el porcentaje de la población entre la edad de 0 y 14 anos $^{12}$. Los resultados oblenidos fueron los siguientes: 


$$
\begin{gathered}
S=18.2928+\underset{(3.496)}{0.01285 \mathrm{Y}}+\underset{(1.629)}{1.59388 \mathrm{G}}-\underset{(5.230)}{1.19645 \mathrm{~F}}-\underset{(1.397)}{0.30253 \mathrm{DA}} \\
\mathrm{R}^{2}=0.669
\end{gathered}
$$

Se puede notar el impacto negativo y significalivo que sobre la tasa de ahorro ejerce el flujo de capital foráneo.

En un importante trabajo para determinar la respuesta macroeconómica de un pais ante el ingreso de capital extranjero. Areskoug postula que dicha respuesta puede consistir de variaciones en el tipo de cambio, en la balanza comercial, en el crédito bancario, en la olerta monetaria o en la lasa de inflación. Los resultados obtenidos de un análisis de 20 paises en vias de desarrollo indican que exisle una asociación positiva entre el ingreso de recursos extemos y la depreciación del tipo de cambio, el incremento del crédito bancario y el incremento en los precios. Asimismo, el autor enconlró que el impacto linal del capilal extranjero depende de la respuesta del país receptor; así, en los países que respondian en términos de la apreciación del tipo de cambio se noló una tendencia a destinar el capital extranjero a la inversión, mientras que cuando la respuesta macroeconómica consislía en incrementar el crédito bancario, los recursos exlernos eran destinados a aumenlos sustanciales en el consumo ${ }^{13}$.

En un trabajo subsecuente Areskoug analiza el impaclo que sobre la formación de capilal lijo, (I), ejerce el flujo de inversión extranjera (F), los otros ingresos de recursos externos (B), y el ingreso nacional ( $Y$ ). Al estimar ecuaciones para cada uno de 21 paises en vias de desarrollo y utilizando dalos anuales del período 1948-1968, encontró que en la mayoria de los casos los coeficientes de las variables $F$ y $B$ no eran significalivos. Encontró, además, que los valores estimados de b difieren considerablemente de país a país, por lo que recomendó precaución al efectuar análisis de corle iransversal. Al comparar las magniludes de los coelicientes asociado con las variables $F$ y $B$, Areskoug infiere que el impacto de la inversión extranjera sobre la inversión tiende a ser mayor que la contribución de los préstamos externos oficiales. El autor concluyó que "la inversión extranjera privada desempena funciones suplementarias y sustitutas en la formación de capital en países en vias de desarrollo. Como en el caso del ingreso de capital oficial, ésta tiende a reducir el ahorro doméslico en el corto plazo, aunque la reducción en el ahorro interno que acompańa el capilal oficial puede ser más pronunciado"14.

Con el fin de delerminar el impacto sobre la tasa de crecimiento económico (G), derivado del ahorro doméstico (S) y de las distintas 
clases de recursos exlernos (ayuda externa (A), inversión privada extranjera $(F)$, y otros recursos externos $(T)$, Papanek estimo una ecuación de corte transversal de una muestra de paises en vias de desarrollo como el período 1950-1970, obteniendo los resultados siguienles: ${ }^{15}$

$$
G=\underset{(2.5)}{1.5}+\underset{(6.0)}{0.20 S}+\underset{(5.8)}{0.39 A}+\underset{(2.5)}{0.17 F}+\underset{(2.1)}{0.19 T} \quad A^{2}=0.37
$$

Estos resultados permitieron al autor concluir que el ahorro doméstico y las distintas clases de recursos extemos explican una tercera parte de la varianza de la tasa de crecimiento económico. Papanek concluyó, además, que el ahorro externo en términos de "ayuda" ejerce un impac10 más signilicativo sobre el crecimiento; sin embargo, encontró que el único componente de los recursos exlernos que mostraba una correlación negativa con el ahorro doméslico era la misma ayuda exlerna, lo que el autor explicó apuntando que esta variable es dirigida precisamente a paises con bajas tasas de ahorro.

John Permazoglu llevó a cabo un estudio econométrico para determinar la asociación entre el crecimiento del produclo doméstico bruto, G, por una parte, y la tasa de crecimiento de la inversión bruta, Gi, y los coeficientes de ahorro interno, Sd, y de ahorro externo Se, por otra ${ }^{16}$. Este autor clasificó la muestra de los paises bajo esludio según su grado de desarrollo: un primer grupo de paises con ingresos per cápita (para el periodo 1955-1966) por debajo de US\$300 dólares a precios constantes (paises A); un segundo grupo de paises con ingresos per cápila entre UIS $\$ 300$ y US $\$ 850$ (paises B) y un tercer grupo con ingresos mayores que US $\$ 850$ (paises $C$ ). Los resullados de las estimaciones econométricas se presentan a continuación.

\begin{tabular}{|c|c|c|c|c|c|}
\hline Grupo de paises & & $\mathrm{Gi}$ & $\mathrm{Sd}$ & $\mathrm{Se}$ & $\mathrm{R}^{2}$ \\
\hline $\mathrm{A}$ & 1.54 & 0.21 & 0.13 & 0.29 & 0.71 \\
& $(1.13)$ & $(0.04)$ & $(0.07)$ & $(0.11)$ & \\
& 3.51 & & 0.05 & 0.57 & \\
$\mathrm{~B}$ & $(1.76)$ & & $(0.11)$ & $0.18)$ & 0.14 \\
& -1.69 & 0.28 & 0.22 & 0.34 & 0.19 \\
& $(0.09)$ & $(0.07)$ & $(0.06)$ & $(0.07)$ & \\
$\mathrm{C}$ & 2.21 & & 0.34 & 0.44 & 0.62 \\
& $(1.73)$ & & $(0.08)$ & $(0.09)$ & \\
& 0.93 & 0.22 & 0.08 & & 0.35 \\
\hline
\end{tabular}

Nota: Las desviaciones estándar se muestran debajo de los correspondientes coeficientes de regresión. 
Los resultados indican que el coeliciente de la variable Gi es más significativo en el caso del grupo de países A y se vuelve insignificalivo en el grupo $\mathrm{C}$.

Por su parte, las variables $S d$ y $S e$ resultan más significalivas en el caso del grupo inemedio de paises.

El autor estimó además, una ecuación para detectar la relación entre las variables Sd y Se, obteniendo los siguientes resultados:

$$
\begin{aligned}
& \text { Grupo A y B: } \quad \operatorname{LogSd}=-1.2285-0.26 \operatorname{LogSe} \quad \mathrm{A}^{2}=0.48 \\
& \text { (0.0285) (0.05) } \\
& \text { Grupo C: } \quad \text { LogSd }=-1.2545-0.26 \text { LogSe } \quad A^{2}=0.72 \\
& \text { (0.0322) (0.05) }
\end{aligned}
$$

Estos resultados confirman la asociación negativa entre el ahorro inlerno y exlerno del coeficiente de ahorro externo de $4 \%$ conducirla a una reducción del coeficiente de ahorro doméstico en $1 \%$.

En un trabajo sobre la relación entre ayuda externa, ahorro y crecimiento económico. Paul Mosley sefiala que los estudios realizados en esta maleria no toman en cuenta la estructura de rezagos entre las entradas de capilal extranjero y las variables dependientes ${ }^{17}$. Senala, además, la necesidad de dififerenciar el impacto de la ayuda externa en dislintos grupos de países clasificados según ingreso per cápila, así como la necesidad de tomar en cuenta la simultaneidad entre el nivel de desarrollo y la ayuda externa por medio de la estimación de ecuaciones simultáneas.

Usando dalos para un grupo de 83 paises en vlas de desarrollo y con datos correspondientes al promedio de los anos setenta, estimo por medio de mínimos cuadrados bietápicos la siguiente ecuación:

$$
Y=\underset{(3.11)}{6.13}-\underset{(1.85)}{0} \underset{(0.59)}{0.94 A}-\underset{(0.43)}{0.023 S} \quad R^{2}=0.0857
$$

en donde $Y=$ tasa de crecimiento promedio del producto interno bruto en el período 1970/77; $A$ = promedio del período 1969-76 de los desembolsos de ayuda concesional como porcentaje del producto (de 1977) del pais receptor; $A X=$ promedio de las olras entradas de recursos externos en el mismo período y como porcentaje del producto; $\mathrm{S}=$ valor en 1977 del ahorro doméstico bruto como porcentaje del producto.

$$
Y=\underset{(3.43)}{5.00}-\underset{(1.74)}{\left(1.08 A^{\prime}-0.34 A X^{\prime}\right.}+\underset{(0.93)}{0.105} \quad A^{2}=0.2885
$$


Se puede notar que al usar métodos de estimación que toman en cuenta la relación simultánea entre crecimiento y ayuda externa, el efecto de la última variable es negativo, como se delectó en otros trabajos, pero su coelicienle no tiene significación estadística. Al utilizar las variables de ayuda extema con un rezago de 5 afios, el coeficiente de determinación aumenta considerablemente, pero ambas variables resullan otra vez sin signiticancia estadíslica.

Con relación a la cuestión de que si la ayuda externa aumenta el consumo, Mosley introduce la consideración de que en aquellos paises que están aumenlando su esfuerzo fiscal, medido como la relación entre ingresos fiscales y producto, los recursos externos no se destinan al consumo. De esa manera presenta la hipótesis de que la influencia posiliva del ahorro externo sobre el crecimiento dependerá de la tendencia en el estuerzo fiscal. Los análisis de la muestra de paises confirman esta hipólesis: en los paies que más ayuda reciben, aquellos con crecimiento economico por debajo del promedio muestran un esfuerzo fiscal declinanle y aquellos con crecimiento acelerado experimentan una tendencia ascendente en el esfuerzo fiscal. Al volver a estimar la ecuación anterior (por mínimos cuadrados bietápicos) usando la variable esfuerzo fiscal, TX, en vez de la tasa de ahorro, los resultados denotan un papel positivo de esta variable y de los recursos externos sobre el crecimiento:

$$
Y=\underset{(0.11)}{-0.26}+\underset{(2.80)}{1.11 A^{\prime}}+\underset{(0.44)}{0.23 A X}+\underset{(3.09)}{0.27 T X} \quad A^{2}=0.3023
$$
76.

en donde TX es el cambio en el esfuerzo fiscal entre 1962/68 y 1972/

Un análisis de las relaciones entre ayuda externa, ahorro doméstico y crecimiento económico en los países asiálicos fue realizado por J.M. Dowling Jr. y U. Hiemenz ${ }^{18}$. Eslos aulores senalan que los distintos estudios que se han elecluado sobre esta temática adolecen de algunas djficultades. Por una parte, el ahorro nacional y el crecimiento económico dependen de varios faclores, en adición a la entrada de capilal extranjero: además, exisle un rezago o período de gestación entre el flujo de capilal y el crecimiento económico que no es tomado en cuenta en la mayoria de trabajos; por olra pare, dado que los paises menos desarrollados tienden a lener más bajas tasas de ahorro y de crecimiento y, a la vez, reciben más ayuda extema, la relación inversa entre crecimiento y recursos externos se puede explicar por este hecho y no por una relación de causalidad macroeconómica. Los autores estiman una serie de ecuaciones de la forma:

$$
\Delta Y=f(F A, K M, S)
$$

En donde: $\Delta Y$ es la tasa de crecimiento real del producto interno bruito; FA es la entrada de ayuda externa, KM es la entrada de capital 
privado; y $\mathrm{S}$ el ahorro doméstico brulo. En adición, los aulores incorporan otras variables explanatorias como la movilización de recursos por parte del sector público, $(T)$, representada por la razón de ingresos fiscales a produclo; el grado de apertura de la economia, (ET), expresado como el cociente de las exportaciones más las importacinnes entre el producto; la participación del sector público en la economia, (E), representado por el gasio público lolal como porcentaje del produclo; y una medida de "represión financiera" (M), medida como la razón de la oferta monelaria ampliamente definida a produclo.

Las ecuaciones lueron estimadas usando datos anuales para los anos selenta en una muestra transversal y algunos de los resultados obtenidos se presentan a continuación:

$$
\begin{aligned}
& \Delta Y=0.47+0.46 \mathrm{FA}+0.72 \mathrm{KM}+0.24 \mathrm{~S} \\
& \text { (2.46) } \quad(2.72 \quad(4.5) \\
& \mathrm{R}^{2}=0.43 \quad \mathrm{DW}=1.96 \\
& \Delta Y=0.86=0.40 \mathrm{FA}+0.71 \mathrm{KM}+0.15 \mathrm{~S}+0.17 \mathrm{~T}+0.03 \mathrm{M} \\
& \begin{array}{llll}
(2.14) \quad(2.78) \quad(2.33) \quad(1.94) \quad(1.80) & 0
\end{array} \\
& R^{2}=0.48 \quad D W=1.94 \\
& \Delta Y=0.25=\underset{(2.14)}{0.43 F A}+\underset{(2.13)}{0.64 K M}+\underset{(3.31)}{0.20 S}+\underset{(1.25)}{0.01 E T}+\underset{(0.50)}{0.03 E} \\
& R^{2}=0.43 \quad D W=1.92
\end{aligned}
$$

En todas estas ecuaciones las variables ayuda externa, capilal privado extranjero y ahorro doméstico son estadísticamente significativas y ejercen un impacto positivo sobre el crecimiento econóimico; las variables que describen la apertura de la economía y la participación económica del sector público resultaron no significativas y aquellas relacionadas con la profundización financiera y la captación de ingresos por parte del sector público resultaron significalivas a un nivel de confianza del $10 \%$.

Estos resullados indicarian que los recursos exlernos ejercen un impacto posilivo sobre el crecimiento económico; sin embargo, cuando las ecuaciones fueron estimadas por minimos cuadrados bietápicos, la relación entre crecimiento y ayuda externa se volvió negativa y el coeficiente de determinación se redujo, a lal grado que la ecuación perdió significancia esladistica.

En adición, los autores estimaron ecuaciones para dos subgrupos: uno que comprende los países asiálicos de rápido crecimiento y olro 
para los palses del Asla Sureste que crecieron menos rápidamente. En el primer grupo la ayuda externa resultó significativa y positiva, y la entrada de capital extranjero resultó negativa, mientras que en el segundo grupo ambas varlables resultaron insignilicativas, como se muestra a continuación:

Palses con rápldo crecimlento:

$$
\begin{gathered}
Y=0.62+\underset{(3.53)}{1.74 F A}-\underset{(1.38)}{0.44 K M}+\underset{(0.40)}{0.05 S}+\underset{(0.23)}{0.02 T}+\underset{(4.37)}{0.09 M} \\
R^{2}=0.39 \quad D W=1.78
\end{gathered}
$$

Paises de lento crecimlento:

$$
\begin{gathered}
Y=0.91+\underset{(1.58)}{0.30 F A}+\underset{(0.42)}{0.55 K M}+\underset{(1.30)}{0.15 S}+\underset{(2.43)}{0.29 T}-\underset{(0.11)}{0.01 M} \\
R^{2}=0.33 \quad D W=2.14
\end{gathered}
$$

Se puede apreciar que la variable prolundización linanciera resulta significativa sólo en el caso de los países de rápido crecimiento y que el esfuerzo fiscal es esladisticamente significativo sólo en los paises de bajo crecimiento.

En un análisis del caso particular de Colombia, Ocampo sehala que existe una diferencia entre ahorro y financiamiento externos, en términos principalmente de los cambios en las reservas internacionales que pueden ocurrir a ralz de un déficit en la cuenla corriente ${ }^{10}$. Este autor investiga el impacto de estas dos variables sobre el ahorro interno público, privado y tolal por medio de técnicas de regresión y encuentra que la relación negativa entre ahorro externo y ahorro doméstico en el periodo de 1950-1969 se explica por el deterioro de los ingresos caleteros. De esa manera, Ocampo concluye que "La relación inversa entre ahorro externo e interno relleja, asi, el uso anticíclico de las finanzan cafeteras y no puede inlerpretarse como una "sustilución" entre una y otra variable".

Conclusiones similares se derivan al emplear la variable financiamiento externo, aunque se nota una relación más estrecha entre el financiamiento externo y el ahorro público que con el ahorro privado. Además, se encuentra un efecto de "desplazamiento" de la tributación ante la disponibilidad de linanciamiento externo: "aún si se tienen encuenta las interrelaciones de política económica mencionadas en el párrato amerior, estos resultados ciertamente evidencian una sustitución entre tributación y financiamiento público externo. Esta sustitución ha sido particularmente fuerte en algunas coyunturas especificas, durante 
las cuales han coincidido un gran dinamismo de la inversión pública y del linanciamiento externo con un debilitamiento de la tributación". Es del caso sefialar que en las ecuaciones econométricas estimadas para el ahorro interno lotal, la variable tasa media de tributación resultó ser estadisticamente significativa.

También se trae a cuenta el reciente estudio de Karras y Levinsohn, que usando datos para el periodo 1960-1982 estimaron funciones de consumo para cada uno de 24 países en vias de desarrollo. La ecuación propuesta expresa el consumo privado $(C)$ en función del ingreso real (Y), el ingreso de capital extranjero (FI) y de variables cualitativas para representar desplazamienlos de las variables Y y FI (DY y DFI respectivamente) ${ }^{20}$. Con la excepción de 2 paises (Brasil y Tunisia) en lodos los países se detectó un impacto positivo del ingreso de capital extranjero sobre el consumo privado.

Tomando en cuenta los resultados que muestran un eleclo negativo del ahorro externo sobre el ahorro doméstico, la pregunta que surge es si existe algún problema de especificación en las ecuaciones estimadas que podrian explicar este comportamiento. Un estudio reciente de Bowles sobre la dirección de causalidad entre las variables ahorro doméstico y ahorro externo, efectuado para 17 paises en vías de desarrollo, enconlró que en 5 países la dirección de causalidad era de ahorro externo a ahorro domésico, en 3 paises la causalidad era a la inversa y en 10 países no se detectaba ninguna relación de causalidad ${ }^{21}$. Se debe sefhalar, sin embargo, que el período de liempo para cada pais abarcó 1961-1981, el cual es sumamente corlo para un estudio de esta naluraleza. De ahí que los resultados deberian de interpretarse con precaución.

Sobre el tema de la causalidad entre inversión, ahorro doméstico y ahorro externo se menciona, además, el estudio de Zaidi para 20 paises en vias de desarrollo el cual encontró que la dirección de la causalidad era de ahorro doméstico a ahorro externo ${ }^{22}$. Este autor reportó que "las pruebas de causalidad apuntan que cambios en el ahorro doméstico y la inversión son las causas y no los efectos de cambios en el saldo de la cuenta corriente", por lo que "los aumentos en el endeudamiento de estos paises reflejan principalmente un intercambio de deuda externa por capital tísico adicional". Se debe nolar que el tamano de las muestras de este estudio fueron entre 25 y 30 afos, periodo relativamente corto para un esludio de causalidad. De esa manera, los resultados pueden ser no contundentes.

En este punto se debe notar que Papanek ha sefialado que la dirección de causalidad entre ahorro externo y ahorro doméstico no es clara precisamente porque bajas tasas de ahorro se correlacionan con altos ingresos de recursos exlernos que ocurren precisamente como una respuesta de los paises donantes para ayudar a los paises más necesila- 
dos. Es decir, no existe una causalidad de ahorro externo a ahorro interno, sino una asociación entre los relativamente allos montos de recursos loráneos que ingresan a paises que por distintas razones experimentan problemas para movilizar recursos domésticos ${ }^{23}$.

En este sentido, es del caso apuntar el interesante trabajo de Rana que sefiala que los estudios sobre el impacto del ahorro externo sobre el ahorro doméstico y el crecimiento económico, no toman encuenta la simultaneidad entre ahorro interno y crecimiento. Para captar la retroalimentación entre estas variables, el autor introduce un sistema de 2 ecuaciones. ${ }^{24}$.

$$
\begin{aligned}
& \mathrm{GR}=A_{0}+A_{1} A+a_{2} F P I+a_{3} S+a_{4} C X+a_{5} C L F \\
& S=a_{6}+a_{7} A+a_{8} F P I+a_{9} C X+a_{10} G D P N+a_{11} G R \\
& \text { en donde }
\end{aligned}
$$

$\mathrm{GR}=$ lasa de crecimiento del producto interno bruto (PIB)

$A=$ Ayuda extranjera como porcentaje del PIB

$\mathrm{FPI}=$ Inversión privada extranjera como porcentaje del IB

$S=$ ahorro doméstico como porcentaje del PIB

$\mathrm{CX}=$ incremento en las exportaciones como porcentaje del PIB.

$C L F=$ incremento en la luerza de trabajo

GDPN = PIB per cápita.

Estas ecuaciones fueron estimadas para los paises asiáticos en vías de desarrolo usando datos anuales para el período 1965-1982. La estimación de la forma reducida del modelo por mínimos cuadrados indirectos permitió calcular las variables estructurales tomando en consideración la simullaneidad de las ecuaciones. Los coeficientes estruclurales representan los efeclos direclos de las variables explicatorias sobre las tasas de horro y de crecimiento económico, a la vez que los coeficientes oblenidos por la estimación de la forma reducida representan los efectos totales de las variables explicativas.

A conlinuación se muestran los electos directos y lotales de la ayuda externa, inversión extranjera y exportaciones sobre la tasa de ahorro y la lasa de crecimienlo del PIB:

\begin{tabular}{lrrrrrrrr}
\hline & \multicolumn{3}{c}{ Efectos direclos } & & \multicolumn{3}{c}{ Efectos lotales } \\
\cline { 2 - 8 } & A & FPI & CX & & A & FPI & CX \\
\hline Tasa de ahorro doméstico & -1.289 & -0.885 & -0.169 & -1.282 & 0.221 & 0.168 \\
Tasa de crecimiento del PIB & 0.134 & 0.666 & 0.193 & 0.008 & 0.688 & 0.210 \\
\hline
\end{tabular}


Se puede nolar que tanto los efectos direclo y tolal de la ayuda exlerna (A) sobre la lasa de ahorro doméstico son negativos; sin embargo, la inversión extranjera (FPI) tiene un impaclo directo negativo sobre el ahorro pero su efecto total es positivo, componamiento contrario al mostrado para las exporlaciones (CX).

Se puede observar, además, que la ayuda exlerna ejerce un electo positivo directo sobre la tasa de crecimiento económico, pero su electo total es sumamenle bajo (0.008). Por su parte, los impactos direclo y lotal de la inversión extranjera sobre el crecimiento son positivos, con valores de alrededor de 3 veces la magnitud de los eleclos de las exportaciones.

Otra critica al modelo de dos brechas se deriva del supuesto de que el ahorro externo no constituye una presión sobre la balanza de pagos en conceplo del servicio de la deuda que dicho ahorro genera. El hecho es que en forma implícita en estos modelos se presupone que lodo el ingreso de capital loráneo es en forma de donación, cle manera que el servicio de la deuda no vendría a constituir un medio para ensanchar la brecha externa. Sin embargo, si se loma en consideración que los recursos externos tienen un costo, surge la posibilidad de que emerjan repercusiones negativas en la balanza de pagos. Considérese, por ejemplo, un ingreso de capilal externo A con una tasa de inlerés $r$ en un pais que tiene una relación incremental producto-capilal k. Si una fracción w de los recursos externos es consumida, el incremenlo en el producto sería de $A(1-w) k$. A su vez, el interés anual pagado por el préstamo $A$, es rA. si se supone además que una fracción $w$ del incremenlo del producto es consumida, el remanenle para atender la obligación exlerna sería A $(1-w) k(1-w)$, lo que debe ser superior a rA para no impaclar negativamente en las cuenlas externas. De esa manera, el ingreso de capilal externo no tendría electos negativos sobre la balanza de pagos si $(1-w)^{2} k$ es superior a la tasa de interés $r^{25}$.

Uno de los trabajos pioneros sobre el papel del endeudamiento exlerno sobre el crecimiento económico es el de Benton F. Massell, quien presentó el siguente modelo ${ }^{26}$ :

$$
\begin{aligned}
Y=C+I+X-M-r D \\
\text { en donde, } \quad Y=\text { producto nacional bruto } \\
C=\text { consumo público privado } \\
Y=\text { inversión } \\
X=\text { exportaciones } \\
M=\text { importaciones } \\
D=\text { saldo de deuda externa } \\
Y=\text { lasa de interés. }
\end{aligned}
$$

El crecimiento de la deuda está dado por la identidad: 


$$
\Delta D=r D+M-X
$$

Y la función de consumo es la siguiente:

$$
C=(1-s) Y
$$

en donde, $s$ es la propensión marginal a ahorrar.

Massell introduce la diferencia entre produclo doméslico brulo, Q,P y el producto nacional bruto $Y$ :

$$
Q=Y+r D
$$

y el incremento de $Q$ es dado por:

$$
\Delta Q=\sigma l \text {, en donde } \sigma \text { es la relación producto-capital }
$$

De las ecuaciones anteriores el aulor deriva la siguienle ecuación para I:

$$
I=s Q+\Delta Q-s r D
$$

Y sustituyendo esta expresión en la ecuación (5) se obliene que:

$$
\frac{\Delta Q}{Q}=Q-\sigma\left(s+\frac{\Delta D-s r D}{Q}\right)
$$

En la relación anterior se puede apreciar que la lasa de crecimiento $Q$ será superior a os si $\Delta D$ es mayor que srD. De esa manera, srD se puede conceplualizar como recursos que se sustraen de los recursos de inversión, o como recursos que se "consumen" en el senlido de que se reslan del ahorro doméstico. De esa manera, el endeudamienlo adicional aumentaría la tasa de crecimiento si srD luese menor que el incremento anual en el endeudamiento.

$$
\text { La expresión } \frac{\Delta Q-\operatorname{srD}}{Q} \text { se puede escribir como: }
$$

$$
\frac{\frac{\Delta Q}{D}-\frac{S r D}{D}}{Q / D}=\frac{D-s r}{Q / D}
$$

Lo que indica que mientras la lasa de crecimiento de la deuda sea superior a sr, los tondos externos aumentarlan la tasa de crecimienlo del producto doméstico bruto. 
Ahora bien, usando la definición de $Y$ dada por la ecuación (4), se puede expresar $\boldsymbol{\Delta} \mathbf{Y}$ asi:

$$
\Delta Y=\Delta Q-r \Delta D
$$

y sustituyendo las ecuaciones (5), (6) y (4) en la ecuación (8), se obliene que:

$$
\begin{aligned}
& \Delta Y=\sigma s Y+\Delta D(\sigma-r) \\
& y Y=\sigma s+(\sigma-r) \frac{\Delta D}{Y}
\end{aligned}
$$

Estas ecuaciones indican que la tasa de crecimiento del producto nacional bruto será superior a os, la tasa dada por Harrod-Domar, si la relación produclo-capilal es mayor que la tasa de inlerés.

Es importante notar que a partir de la ecuación (7) se puede calcular el impacto sobre la lasa de crecimiento $O$ de un incremento $\Delta r$ de la tasa de interés sobre su tendencia hislórica, ro:

$$
\begin{aligned}
Q & =\sigma\left(s+\frac{(\Delta D-(r o+\Delta r) s D}{Q}\right. \\
& =\sigma s+\sigma \frac{(\Delta D-s r o D-s \Delta r D)}{Q} Q \frac{Q}{Q}
\end{aligned}
$$

Se puede apreciar que el incremento de la lasa de interés disminuye el crecimiento del producto en $\operatorname{sr} \frac{D}{Q}$, y que el monto absoluto de la reducción del producto doméstico bruto es dado por:

$$
\Delta Q=-\sigma S D \Delta r, \text { por lo que el multiplicador es el siguiente: }
$$

$$
\frac{\Delta \mathrm{Q}}{\Delta \mathbf{r}}=-\sigma \mathrm{SD}
$$

Un modelo preciso para analizar el impacto del capital extemo en el crecimiento económico tomando en cuenta el servicio de la deuda ha sido propuesto por Wasow ${ }^{27}$. Este aulor presenta las siguienles ecuaciones: 


$$
\begin{aligned}
& Y=Q-\theta \\
& \dot{Q}=\sigma I \\
& I=S+F \\
& S=a+b Y+c F \\
& \dot{\theta}=i F
\end{aligned}
$$

En donde: $Y=$ producto nacional bruto

$$
\begin{aligned}
& \dot{Q}=\text { producto interno bruto } \\
& \dot{\theta}=\text { pago de faclores al exterior } \\
& \mathbf{I}=\text { inversión } \\
& \mathbf{S}=\text { ahorro doméstico } \\
& \mathbf{i}=\text { tasa de interés sobre capital externo } \\
& \sigma=\text { relación producto-capital }
\end{aligned}
$$

Además, un punlo $(\bullet)$ sobre la variable representa su derivada con relación al tiempo.

El sistema de ecuaciones se puede traducir en una ecuación diferencial básica:

$$
\begin{aligned}
\dot{Y} & =\dot{Q}-\dot{\theta} \\
& =\sigma 1-i F \\
\dot{Y} & =\sigma(a+b Y+c F+F)-i F \\
& =\sigma a+b Y+(\sigma(1+c)-i) F
\end{aligned}
$$

Esta ecuación diferencial se puede resolver para $Y$ suponiendo diferentes trayectorias para $F$.

De la ecuación anterior se puede encontrar la contribución del capital externo al crecimienlo económico:

$$
\frac{\delta Y}{\delta F}=\sigma(1+c)-1
$$

Se debe notar que el ahorro externo propulsa el crecimiento económico sólo si se cumple la condición $(\sigma(1+c) 0$, > o sea si $\sigma(1+c)>i$. Un punto importante es que para valores razonables de $\sigma, c e i$, esla condición se puede cumplir al menos que c lenga un valor negativo muy alto. 
En todo caso, cuando el coeficiente c tiene un valor negalivo la contribución del capital extranjero al crecimiento se reduce considerablemente.

Por otra parte, en este punto se debe senalar que los modelos resenados anteriormente no toman en cuenta la posibilidad de dismluir las brechas de ahorro y de importaciones mediante incrementos en la producción exportable o en el ahorro de los sectores público o privado. En la primera parte de este trabajo se expresó la brecha de ahorro como la expresión:

$$
g=\frac{s}{\alpha}+\frac{1}{\alpha}
$$

En el Gráfico No. 1 se presenta la trayectoria del crecimiento económico, $g$, en función del ahorro externo como proporción del ingreso, $f$. La brecha externa se represemla como una línea con una ordenada en el origen igual a $\frac{s}{\alpha}$ o sea el crecimienlo que se obtendría en a ausencla de ahorro externo.

A la vez, en la identidad de la brecha externa $E+F=M$, las importaciones totales se pueden desglosar en importaciones de capital Mk y de otros bienes Mo y se puede suponer que Mo es una función del ingreso (Mo $=m Y$ ), en donde $m$ es la proporción marginal a importar). Mk, a su vez, da lugar a un incremento del producto $M k=\alpha, \Delta y$, en donde $\alpha$, es la relación capilal-producto asociada a los bienes de capital importados.

Enionces la brecha externa se puede representar así:

$$
\begin{aligned}
E+F= & M k+m Y \\
E+F= & \alpha^{\prime} \Delta Y+m Y \\
& Y \text { dividiendo por } Y: x+1=\alpha^{\prime} \frac{\Delta Y}{Y}+m \\
& Y \text { resolviendo por } \frac{\Delta Y}{Y}=\frac{x-m+f}{\alpha^{\prime}}=g
\end{aligned}
$$

Esta expresión se representa en el Gráfico 1 como una linea con un intercepto en la abcisa igual a $m-x$ y una pendiente igual a $1 / \alpha$. 


\section{Graflce No. 1}

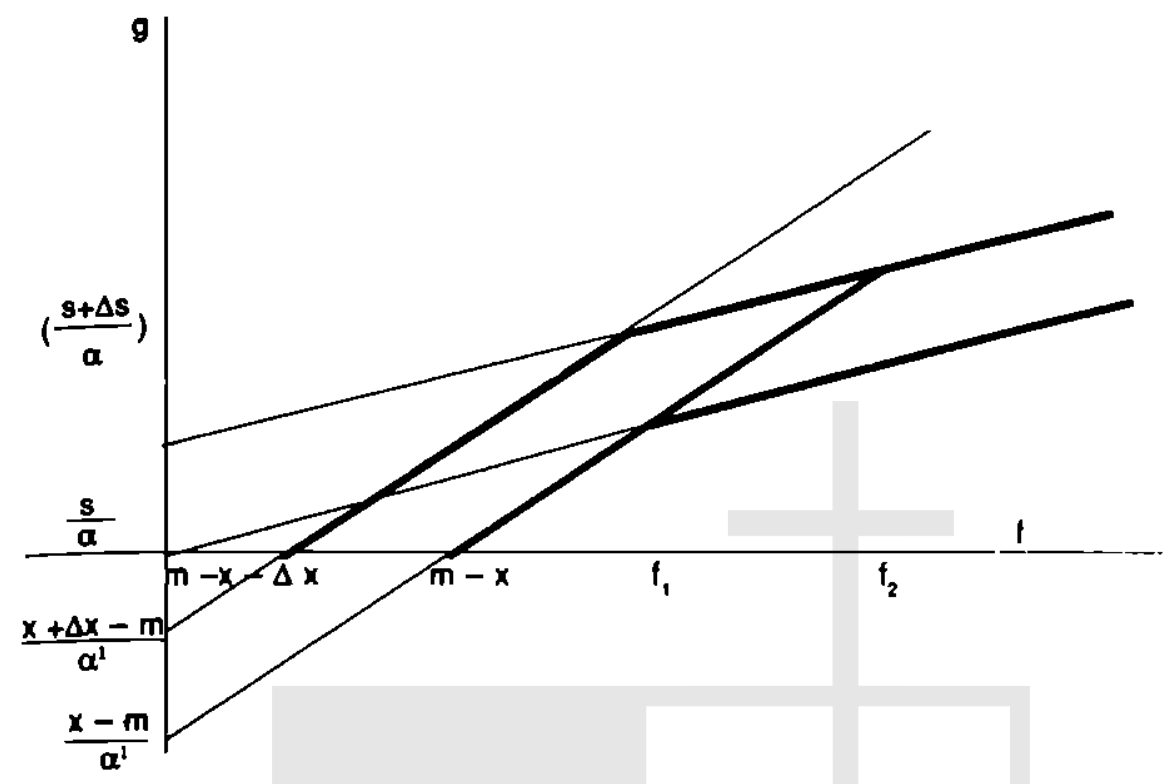

La posibilidad de cambio estruclural se puede representa suponiendo que mediante esfuerzos internos la tasa de ahorro crece en $\Delta s$, de manera que la linea de la brecha de ahorro tiene ahora una ordenada en el origen igual a $(s+\Delta s) / \alpha$. En este caso se puede notar que para valores de $f$ superiores a $f$, el crecimiento es superior al caso anterior, ya que la trayectoria de $\mathrm{g}$ está dada por la línea de la brecha de comercio hasta $\mathbf{t}_{2}$. Es decir, tasas más altas de crecimiento se oblienen en los mismos valores de $\mathrm{l}$. Si se supone además que el coeliciente de las exportaciones crece en $\Delta x$, la línea de la brecha de comercio se mueve hacia la izquierda y tasas más altas de crecimiento se oblienen a los mismos valores de I. Es decir, la posibilidad de crecimiento se expanden cuando la economía aumenta su tasa de ahorro o de exportaciones, o disminye su tasa de importaciones.

Una limitante que se nota en todos los trabajos es que son de una naturaleza estática, sin tomar en cuenta los electos en el presente de las trayectorias pasadas del endeudamiento externo. Es decir, se postulan relaciones entre ahorro doméstico y exlerno sin considerar como su relación en el presente es influenciada por el comportamiento pasado de estas variables. Para tener una apreciación de las relaciones dinámicas entre estas variables, se puede formular la función de importaciones, $M$, 
de acuerdo a una estructura dinámica:

Ya que la demanda por recursos exlernos $F$ está dada por $F=M-E$, en donde $E$ representa las exportaciones, ésta se puede escribir:

$$
F=b l+\mathrm{CM}_{-1}-\mathrm{E}
$$

y dado que $M_{-1}=F_{-1}+E_{-1}$, la ecuación para $F$ se puede escribir así:

$$
F=b l+c F_{-1}+\left(\frac{c}{1+V}-1\right) E
$$

en donde $\mathrm{V}$ es la tasa de crecimiento de las exportaciones Si la expresión $\left(\frac{C}{1+V}-1\right)$ es representada por $w, y$ dado que $I=\frac{\Delta Y}{\alpha}$. en donde $\Delta Y$ es el incremento en el ingreso y $\alpha$ es la relación productocapital, sustiluyendo en la expresión anlerior se obtiene:

$$
F=\frac{b \Delta Y}{\alpha}+C F+w E
$$

y dividiendo por $Y$ :

$$
f=\frac{b}{\alpha} g+\frac{c t-1}{(1+g)}+w e
$$

en donde $g=\frac{\Delta Y}{Y}=$ lasa de crecimiento de $Y$

$$
I=\frac{F}{Y} \quad e=\frac{E}{Y}
$$

Si se supone que g y $\mathrm{w}$ son constantes, la ecuación anterior se puede escribir:

$$
\begin{aligned}
f & =\frac{b}{\alpha} g+\frac{c}{1+g} f_{-1}=w e \\
& =\frac{b}{\alpha} g+w e+\frac{c b}{\alpha(1+g)} g+\frac{c w e}{(1+g)}+\frac{c^{2}}{(1+g)} \frac{f^{2}}{(1+g)} f_{-2}
\end{aligned}
$$




$$
\begin{aligned}
& =\frac{b}{\alpha} g+w e+\frac{c b}{\alpha(1+g)} g+\frac{c w e}{(1+g)}+\frac{c^{2}}{(1+g)(1+g)}\left(\frac{b g}{\alpha}+\right. \\
& \left.\quad+w e+\frac{c}{1+g} t_{-3}\right)
\end{aligned}
$$

De esta manera se puede notar que la demanda de recursos exlernos está influenciada por la tasa actual y la tasa histórica de crecimiento económico.

Bajo esta especilicación, la influencia de las expontaciones sobre 1 está dada por:

$$
\begin{aligned}
\frac{\delta f}{\delta \theta}= & W\left(1+\frac{c}{(1+g)}+\frac{c^{2}}{(1+g)(1+g)}\right. \\
& \left.+\frac{c^{3}}{(1+g)(1+g)} \frac{}{(1+g)}+\ldots\right)
\end{aligned}
$$

o sea:

$$
\frac{\delta 1}{\delta e}=W\left(1+=\Sigma, n=1 \frac{c}{(1+g)}\right)
$$

En la expresión anterior nólese que ante una especificación dinámica de la función de importaciones, el impacto directo de las exportaciones sobre el ahorro externo ha disminuido, de manera que un monto mayor de exportaciones se requeririan para ocupar el lugar de una unidad de recursos externos. Además, existe un impacto sobre la demanda de recursos externos 1 provenientes de las exportaciones pasadas. Así, en un ano dado la demanda de recursos externos obedece a eventos propios de ese ano $y$. además, a los niveles de exportaciones de afios pasados. De ahi que cada ano se requeririan mayores montos de exporlaciones para cerrar la brecha externa que a la vez se ensancha por el efecto de arrastre de las exportaciones históricas. De allí que sería inexacto cuantificar la relación entre ahorro interno y externo en un contexto estático. Esto es más aparente al tomar en cuenta la relación que existe entre el ahorro doméslico y la tasa de crecimiento económico, 10 que indica que en la expresión anterior existiria una relación de simultaneldad de $f$ con la tasa de ahorro interno.

\section{Consideraclones finales}

De la resefia anterior sobre las relaciones entre ahorro externo e in- 
terno no se puede concluir categóricamente que existe un efecto negativo de los recursos externos en la formación del ahorro doméstico. Los problemas de simultaneidad y de causalidad todavía no están resueltos, ni siquiera suficientemente identificados, para poder discernir con claridad y en forma general el efecto depresivo del ahorro externo De allí surge la necesidad de analizar el impacto de los recursos externos en un marco más amplio que permita lomar en cuenta los distintos componenles macroeconómicos que dan origen a la demanda por capital extranjero, la relación entre las imporlaciones de bienes de capital y el crecimiento económico $y$, en particular, los efectos dinámicos de la inversión y las exportaciones sobre la demanda de recursos externos.

\section{REFERENCIAS}

1. El modelo más conocido es el de Hollis B. Chenery y Allan Strout, "Foreign Assislance and Economic Developmenl", American Economic Review, vol. 56, 1966, pp. 679-733. Véase también, Ronald I. Mckinnon, "Foreign Exchangenomic Development and Efficient Aid Allocation", Economic Journal, vol. 74. 1964, pp. 388-409. Un trabajo precursor fue el de P.N. Rosenstein-Rodan, "International Aid for Underdeveloped Countries", Review of Economics and Statistics, vol. 43, mayo 1961, pp. 107-138. Aplicaciones a nivel de palses se encuentran en Jaroslav Vanek. Estimating Foreign Resources Needs for Economic Development, New York, McGraw HII Co., 1967; Irma Adelman y Hollis Chenery, "Foreign Aid and economic Development; The Case of Greecen", Review of Economics and Statistics, vol. 48, 1966, pp. 1-19; ILPES. La Brecha Comercial y la Integración Latinoamericana, México, Siglo XXI Editores, 1967. Una excelente reformulación del modelo de las dos brechas se encuentran en Edmar Bacha, "Crecimienlo con Olerta Limitada de Divisas: Una Revaluación del Modelo de Dos Brechas", en El Milagro y la Crisis, Edmar Bacha, ed., México, Fondo de Cultura Económica, pp. 374-394.

2. Es en la temprana literatura de la CEPAL en donde se encuentran las primeras relerencias a las brechas internas y externas. Véase Celso Furtado, Development and Underdevelopment, Berkely, University ol California Press, 1964.

3. Keith B. Grilfin, "Foreign Capilal, Domestic Savings and Economic Development, Bulletin of the Oxford University Institute of Economics and Statistics, vol. 32, No. 2, mayo 1970, pp. 99-112.

4. Keith B. Griflin y John L. Enos, "Foreign Assistance; Objectives and Consequences", Economic Development and Cultural Change, vol. 18, No. 3, abril 1970, pp. 313-326.

5. M. A. Rahman, "Foreign Capital and Domestic Savings: A Test of Haavelmo's Hypothesis with Cross-Country Data", Review of Economics and Statistics, vol. 50, No. 1, 1968.

6. Thomas E. Weisskopl, "The Impact of Foreign Capital Inflow on Domestic Savings in Underdeveloped Countries", Journal of International Economics, 
vol. 2, 1972, pp. 25-38. Weisskopt también estimó ecuaciones para 17 palses en vias de desarrollo y en 10 de ellos el coeliciente de F resultó ser negalivo. Los resultados para los países centroamericanos comprendidos en el trabajo fueron los siguientes:

Costa Rica: $\quad-60.0+0.342 Y-0.584 F \quad+0.072 E \quad R^{2}=0.972$ (2.54) (2.83) (1.25) (0.17)

Honduras: $\quad 5.6+0.067 \mathrm{Y}-0.880 \mathrm{~F}+0.271 \mathrm{E} \quad \mathrm{A}^{2}=0.999$ $(1.10) \quad(2.14) \quad(2.40) \quad(2.62)$

(Los estadisticos $t$ se muestran entre paréntesis debajo de los respectivos coeficientes en éstas y otras ecuaciones presentadas en las referencias).

7. Luis Landau, "Saving Functions for Latin America", en Studies in Development Planning, Hollis Bl. Chenery, editor, Cambridge, Harvard University Press, 1971, pp. 299-321.

8. Las estimaciones del coeliciente b para los paises centroamericanos comprendidos en el estudio y sus estadísticos $\mathrm{t}$, se presentan a continuación:

$\begin{array}{lcc} & b & 1 \\ \text { Costa Rica } & -0.62 & 9.0 \\ \text { El Salvador } & -060 & 5.5 \\ \text { Guatemala } & -0.51 & 3.2 \\ \text { Honduras } & -0.40 & 2.5 \\ \text { Nicaragua } & -0.56 & 4.3\end{array}$

Se puede nolar que en lodos los palses el impacto del ahorro externo sobre el ahorro doméstico resultó negativo y estadísticamente significativo.

9. Hollis B. Chenery y Peter Eckstein, "Development Alternatives for Latin Americas" Journal of Political Economy, vol. 78. No. 4, julio/agosto 1970. La ecuación estimada es de la forma $S=a+b Y+c E+d F$, en

donde: $\quad S=$ ahorro doméstico

$Y=$ producto nacional bruto

$E$ = exportaciones

$F=$ ahorro externo

Los resultados para los palses centroamericanos son los siguientes:

$\begin{array}{lcccccr} & \mathbf{a} & \mathbf{b} & \mathrm{c} & \mathbf{d} & \mathbf{A} \\ \text { Costa Rica } & 16.5 & 0.05 & 102.0 & -0.26 & 0.17 \\ & (1.56) & (1.23) & (0.46) & (0.47) & \\ \text { El Salvador } & -18.2 & 0.08 & 173.0 & -0.95 & 0.88 \\ & (1.42) & (3.12) & (1.39) & (9.96) & \\ \text { Guatemala } & \mathbf{\theta 8 . 4} & 0.07 & \mathbf{8 7 0 . 0} & 0.22 & 0.69 \\ & (4.40) & (2.70) & (3.31) & (0.94) & \\ \text { Honduras } & -38.3 & 0.10 & 254.0 & -0.25 & 0.88 \\ & (3.33) & (3.72) & (3.50) & (1.08) & \\ \text { Nicaragua } & -39.4 & 0.16 & 143.0 & -1.09 & 0.78 \\ & (5.89) & (3.95) & (2.34) & (4.58) & \end{array}$


Estos resultados indican que sólo para El Salvador y Nicaragua los coeficientes del ahorro externo resultaron significativos.

10. Luis René Cáceres, "Domeslic Saving and Investment in Central America and the Role of the Central American Bank Ior Economic Integration", Savings and Development, No. 3, 1985, pp. 265-296. Las ecuaciones estimadas son las siguientes:

\begin{tabular}{|c|c|c|c|c|c|}
\hline Guatemala & $S=$ & -40.02 & $\begin{array}{c}0.26 \mathrm{Y} \\
(5.84)\end{array}$ & $\begin{array}{c}-0.40 \mathrm{~A} \\
(2.22)\end{array}$ & $\mathrm{A}^{2}=0.93$ \\
\hline El Salvador & $\mathbf{S}=$ & 4.13 & $+\begin{array}{c}0.15 Y \\
(5.84)\end{array}$ & $\begin{array}{c}-0.70 \mathrm{~A} \\
(2.22)\end{array}$ & $\mathrm{A}^{2}=0.60$ \\
\hline Honduras: & $\mathbf{S}=$ & 11.13 & $\begin{array}{c}0.17 Y \\
(6.47)\end{array}$ & $\begin{array}{c}-0.17 \mathrm{~A} \\
(0.21)\end{array}$ & $\mathrm{A}^{2}=0.83$ \\
\hline Nicaragua & $S=$ & 23.84 & $\begin{array}{c}+0.14 Y \\
(4.81)\end{array}$ & $\begin{array}{c}-0.47 \mathrm{~A} \\
(3.22)\end{array}$ & $\mathrm{A}^{2}=0.49$ \\
\hline Costa Rica & $S=$ & -35.30 & $\begin{array}{c}+0.22 Y \\
(21.00)\end{array}$ & $\begin{array}{c}-0.59 \mathrm{~A} \\
(5.64)\end{array}$ & $\mathrm{R}^{2}=0.99$ \\
\hline
\end{tabular}

11. Contantin Voivodas, "Export, Foreign Capital Inflow, and South Korean Growth", Economic Development and Cultural Cange, vol. 22, No. 3, abril 1974, pp. 480-484.

12. Kahlaya L. Gupla, "Foreign Capital Inflows, Dependency Burden, and Saving Rates in Developing Countries A Simultaneous Equation Model", Kyklos, vol. 28, 1975, pp. 358-374.

13. Kaj Areskoug. "Foreign Capital Utilization and Economic Policies in Developing Countries", Review of Economics and Statistics, vol. 55, No. 2, mayo 1973, pp. 182-189.

14. Kaj Areskoug. "Private Foreign Investment and capital Formation in Developing Economic Development and Cultural Change, vol. 24, No. 3, abril 1976, pp. 539-547. se preenlan a continuación los resultados para los países centroamericanos comprendidos en este estudio:

$\begin{array}{llrrrr}\text { Gualemala } & I= & -1.22 \mathrm{~F} & +0.75 \mathrm{~B} & +0.08 \mathrm{Y} & \mathrm{A}^{2}=0.72 \\ & & (0.83) & (0.56) & (0.06) & \\ \text { El Salvador } & I= & 0.01 \mathrm{~F} & -0.36 \mathrm{~B} & -0.20 \mathrm{Y} & \mathrm{R}^{2}=0.90 \\ & & (0.45) & (0.60) & (0.03) & \end{array}$

15. Guistav F. Papanek, -Aid, Foreign Privale Investment, Savings, and Growth in Less Developed Countries", Journal of Political Economy, vol. 81, No. 1. enero/febrero 1973, pp. 120-130.

16. John Pesmazoglu, "Growth, Investmen and Savings Ratios: some Long and Medium Term Associations by Group of Countries", Bulletin of the Oxford University Institute of Economics and Statiscts, vol. 34, No. 4, Noviembre 1972, pp. 309-328.

17. Paul Mosley, "Aid, Savings and Growth Revisited", Oxford Bulletin of Economics and Statistics, Vol. 42, No. 2, Mayo de 1980, pp. 79-96.

18. J. M. Dowling Jr. y H. Hilmenz, Aid Savings and Growth in the Asian Region", The Developing Economics, Vol. 21, No. 1. Marzo de 1983, pp. 313. 
19. José A. Ocampo, "Una nota sobre la Relación enlre el Financiamiento Externo, Ahorro e Inversión", Ensayos sobre Politica Económica, No. 13, junio 1988, pp. 87-97.

20. Homi J. Kharas y Jim Levinshon, "LDC Savings Rates and Debt Crisis", World Development, vol. 16, No. 7, julio 1988, pp. 779-786. Para Costa Rica y Nicaragua, los dos palses centroamericanos comprendidos en el estudio, los resultados fueron los siguientes:

\begin{tabular}{|c|c|c|c|c|c|}
\hline $\begin{array}{l}\text { Costa Rica: } \\
\text { Nicaragua: } \\
\text { 1962-1982 }\end{array}$ & $\begin{array}{l}C= \\
C=\end{array}$ & $\begin{array}{c}0.693 \\
(9.91) \\
0.513 \\
(2.12)\end{array}$ & $\begin{array}{l}+0.615 Y \\
(28.4) \\
+0.602 Y \\
(0.855)\end{array}$ & $\begin{array}{c}+0.623 \mathrm{FI} \\
(5.91) \\
+0.334 \mathrm{FI} \\
(1.93)\end{array}$ & $D . W .=1.2 \theta$ \\
\hline & & DY & $\begin{array}{c}+0.693 \text { DFI } \\
(1.43)\end{array}$ & & $D \cdot W .=1.12$ \\
\hline
\end{tabular}

21. Paul Bowles, "Foreign Aid and domestic Savings in Less Developed Countries: Some Tests for Causality", World Development, vol. 15, No. 6, 1987, pp. 789-796.

22. Igbal Mehdi Zaidi, "Saving, Investment, Fiscal Delicit, and the External Indebiness of Developing Countries", World Development, vol. 13, No. 5 , mayo 1985. pp. 573-588.

23. Gustav F. Papanek, -The Effect of Aid and Other Resource Transfers on Savings and Growth in Less Developed countries", Economic Journal, vol. 82, No. 327, sepliembre 1972, pp. 934-950.

24. Pradumna B. Rana, "Foreign Capital, Exports, Savings and Growth in the Asian Region", Savings and Development, vol.' 11, No. 1, 1987, pp. 5-28.

25. Este punto se discule en Keith B. Griflin International Ineguality and National Poverty, New York, Holmes and Meier Publishers Inc., 1978, capílulo 3. Véase también, A. Qayun, "Economic Criteria for Foreign Loans", Economic Jorunal, junio 1966 y Deepak Lal, "The Evaluation of Capital Inllows", Industry and Development, No. 1. 1978, pp. 2-19.

26. Benton F. Massell, "Exports, Capital Imports and Economic Growth", Kyklos, No. 4, 1964, pp. 626-635.

27. Bernard Wasow, "Saving and Dependence vith Externally Financed Growth", Reviwe of Economics and Statistics, vol. 61, No. 1. febrero 1979, pp. 150-154. Véase lambién de este autor, "Dependent Growth in a Capital Importing Economy: The Case of Puerto Rico", Oxford Economic Papers, vol. 30, marzo 1978, pp. 117-129. 